\title{
A new spectroscopic and eclipsing binary BD -20 4369
}

\author{
A.S. Kravtsova ${ }^{1}$, I.M. Volkov ${ }^{1,2}$ and T. Pribulla ${ }^{3,4}$ \\ 1 Sternberg Astronomical Institute, Lomonosov Moscow State University, \\ Universitetskij Ave. 13, 119992 Moscow, Russia \\ 2 Institute of Astronomy, Russian Academy of Sciences, Pyatnitskaya Str., 48, \\ 109017 Moscow, Russia, (E-mail: hwp@yandex.ru) \\ 3 Astronomical Institute of the Slovak Academy of Sciences \\ 05960 Tatranská Lomnica, The Slovak Republic \\ 4 ELTE Gothard Astrophysical Observatory, 9700 Szombathely, Szent Imre \\ h.u. 112, Hungary
}

Received: October 31, 2019; Accepted: January 5, 2020

\begin{abstract}
Absolute physical and geometrical parameters of the system are derived. BD-20 4369 is a strongly evolved stellar system after the first mass exchange.
\end{abstract}

Key words: stars: individual: BD-20 4369 - stars: eclipsing binaries - methods: observational - techniques: spectroscopy, photometry

\section{Introduction}

BD -20 4369 was found to be an Algol-type eclipsing binary from the 2007 MOST photometry (see Pribulla et al., 2010). Due to its southern location it is rather difficult to obtain precise photometrical data from the northern hemisphere. The shallow secondary minimum occurs at phase 0.5 indicating a circular orbit for this detached binary. BD-20 4369 was observed by the ASAS program, but was not identified as a variable.

\section{Observations and data reduction}

Photometric observations were made during 2015-2019 in Simeiz Observatory of INASAN on Mt. Koshka (Crimea). We used a FLI PL09000 CCD camera with a $B V R c I c$ filter set mounted on a 1-m reflector, and a VersArray512UV with $U B V R I R c$ filters on a 60 -cm telescope. We analyzed the photometric data using the methods described in our earlier work (Volkov \& Volkova, 2009; Volkov et al., 2017).

High-resolution echelle spectra were obtained at the LCO observatory in Chile during 2008. Spectra were analyzed using the broadening-function tech- 
nique developed by Rucinski (1992). The radial velocity curve is presented in Fig. 1.

Here we present the analysis of our $V$ observations combined with the radial velocity curve. Observations in other bands are not completed yet and should be continued in the future.

The light curve in $V$ phased with the ephemeris

$$
\mathrm{HJD}(\mathrm{Min})=2453321.01010(5)+3.099035(1) \times E
$$

is presented in Fig. 1. The best solution for the photometric $V$ observations was found for a very large level of third light, $L_{3}=0.55$. This fact should be explained by future investigations as we did not find the lines of the third component in our spectra. The values of the derived parameters of the system are presented in Table 1. The obtained photometric parallax is close enough to the $G A I A$ value $\pi=00^{\prime \prime} 0055(2)$. BD -20 4369 is a strongly evolved stellar system after the first mass exchange.

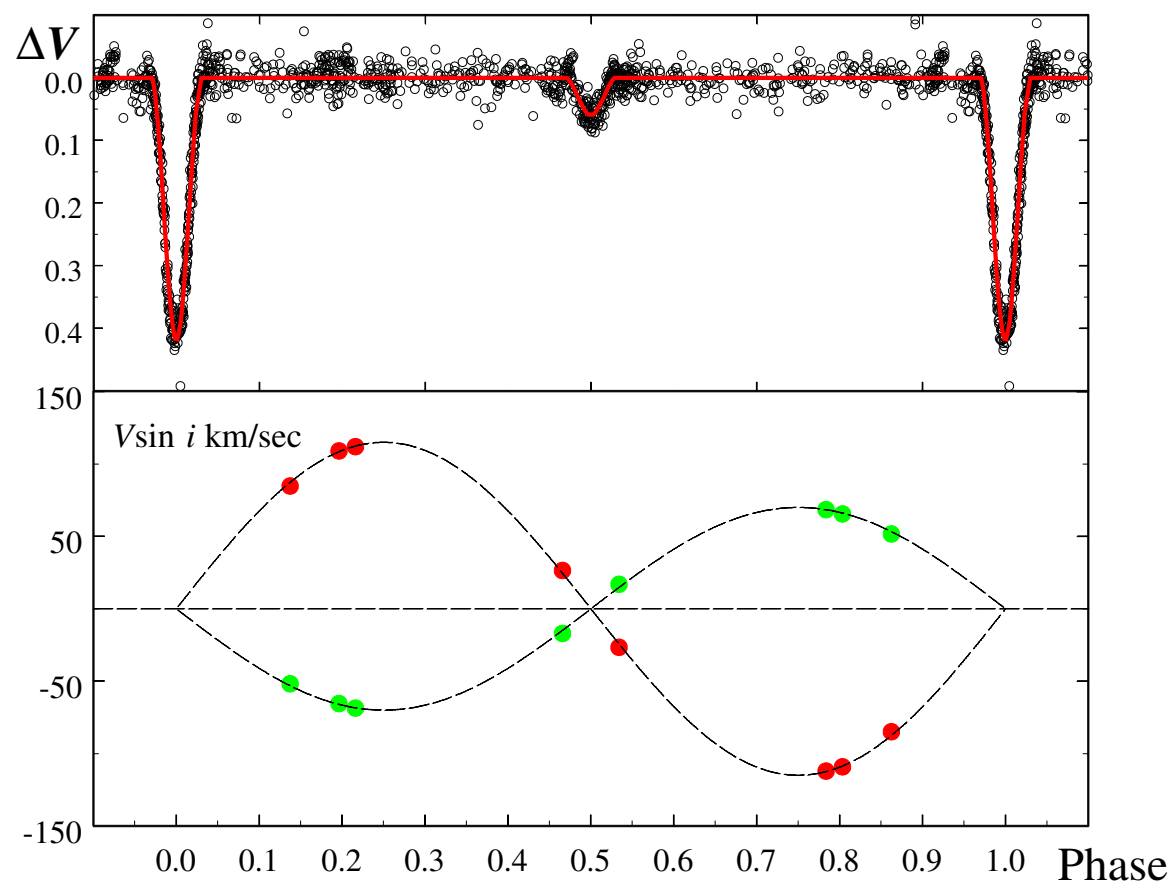

Figure 1. Upper panel: Light curve in $V$ of BD -20 4369, with the red line representing the model. Lower panel - the radial velocities curve, $V_{\gamma}=-21.1 \pm 0.2 \mathrm{~km} / \mathrm{s}$ been moved to the zero point. 
Table 1. The parameters of the BD-20 4369 system

\begin{tabular}{lll}
\hline \hline Parameter & Primary & Secondary \\
\hline \hline$K\left[\mathrm{~km} \mathrm{~s}^{-1}\right]$ & $70 \pm 1.5$ & $115 \pm 1.4$ \\
$M / M_{\odot}$ & $1.27 \pm 0.02$ & $0.80 \pm 0.015$ \\
$R / R_{\odot}$ & $0.83 \pm 0.03$ & $1.38 \pm 0.04$ \\
$\log g$ & $4.71 \pm 0.02$ & $4.04 \pm 0.02$ \\
$T[\mathrm{~K}]$ & $6600 \pm 100$ & $4770 \pm 80$ \\
$E(B-V)$ & $0.20 \pm 0.04$ \\
Inclination, $i\left[^{\circ}\right]$ & $87.6 \pm 0.1$ \\
Semi-major axis, $a\left[R_{\odot}\right]$ & \multicolumn{2}{c}{$11.3 \pm 0.2$} \\
Parallax, $\pi\left[^{\prime \prime}\right]$ & \multicolumn{2}{c}{$0.0057 \pm 0.0004$} \\
\hline \hline
\end{tabular}

Acknowledgements. This work is partially supported by RFBR research project 18-502-12025, RNF grant 14-12-00146 (IMV), a scholarship of the Slovak Academic Information Agency (SAIA) (ASK, IMV), by the VEGA grant of the Slovak Academy of Sciences No. 2/0031/18 (TP) and by the Slovak Research and Development Agency under the contract No. APVV-015-458 (TP). IMV appreciate the support from the Program of development of M.V. Lomonosov Moscow State University (Leading Scientific School 'Physics of stars, relativistic objects and galaxies').

\section{References}

Pribulla, T., Rucinski, S. M., Latham, D. W., et al., Eclipsing binaries in the MOST satellite fields. 2010, Astronomische Nachrichten, 331, 397, DOI: 10.1002/asna.201011351

Rucinski, S. M., Spectral-line broadening functions of WUMa-type binaries. I AW UMa. 1992, Astron. J., 104, 1968, DOI: 10.1086/116372

Volkov, I. M., Chochol, D., Grygar, J., Mašek, M., \& Juryšek, J., Orbital period changes in RW CrA, DX Vel and V0646 Cen. 2017, Contributions of the Astronomical Observatory Skalnate Pleso, 47, 29

Volkov, I. M. \& Volkova, N. S., The physical parameters and orbit of the eclipsing binary system GSC $45961254=$ SAO 3282. 2009, Astronomy Reports, 53, 136, DOI: 10.1134/S106377290902005X 